\title{
Inestabilidad Cromosómica Inducida por el Inhibidor de la Proteína Quinasa Aurora A, MLN8237.
}

Investigador principal: Ana Maritza Erazo Milla, MSc., Universidad Nacional Autónoma de Honduras (UNAH)

Asesora: Katherine Marcelaine, PhD., Universidad de Chile

Colaboradores: Ignacio Maureira, Victor Inostroza, estudiantes de Tecnología Médica, Universidad de Chile.

Investigación desarrollada en la Facultad de Medicina, Universidad de Chile; Santiago, Chile.

\section{RESUMEN:}

\section{Justificación:}

La proteína quinasa Aurora-A (AURKA) es fundamental para el ensamblaje y formación del huso mitótico. Su sobre-expresión o amplificación se ha visto asociada a varios tipos de cánceres, lo que la ha convertido en un blanco de interés terapéutico; esto ha llevado al desarrollo de diversas moléculas inhibidoras de la actividad de esta quinasa. Una de estas moléculas, MLN8237, está siendo evaluada en diversos estudios clínicos, como tratamiento para tumores sólidos y hematológicos. Los estudios in vitro y en modelos animales muestran que estos inhibidores inducen aneuploidía y muerte celular. Por su parte, los estudios clínicos iniciales muestran promisorios resultados relativos a la progresión tumoral. Sin embargo, pese al inminente uso de estos inhibidores en un corto o mediano plazo como terapia anti-tumoral, el impacto de la inhibición de AURKA en células no tumorales ha sido pobremente documentado.

\section{Objetivo:}

En este trabajo se evaluó el efecto de MLN8237 sobre la apoptosis y la frecuencia de aberraciones cromosómicas estructurales y numéricas, en linfocitos de sangre periférica provenientes de individuos sanos.

\section{Métodos:}

Linfocitos de 8 individuos fueron cultivados in vitro y tratados con MLN8237 a concentraciones de $0.1,0.5$ y $1 \mu \mathrm{M}$ por 3 a 5 días. La apoptosis se cuantificó mediante citometría de flujo y las aberraciones cromosómicas se determinaron a través de análisis y recuentos de estructuras cromosómicas en placas metafásicas. 


\section{Resultados:}

Los resultados indican que MLN8237 induce apoptosis dependiente de la dosis pero no del tiempo de exposición. En las distintas condiciones evaluadas, el inhibidor no indujo aberraciones cromosómicas estructurales. Sin embargo, existió un aumento significativo de las poliploidías y aneuploidías que fue mayor, a mayor tiempo de exposición.

\section{Conclusión:}

Estos resultados sugieren que las células no tumorales que sobreviven al inhibidor pueden adquirir mutaciones cromosómicas numéricas que podría tener un impacto en el desarrollo de neoplasias secundarias al tratamiento.

\section{CORRESPONDENCIA:}

Ana M. Erazo:

Departamento de Biología, Edificio J1, 3er piso, Ciudad Universitaria, Boulevard Suyapa, Tegucigalpa MDC, Francisco Morazán, Honduras.

Tel. Oficina (504)-232-2110 ext. 170

Tel. Casa (504)-2236-7711

Tel. Celular (504)-9505-2793

ana.erazo@unah.edu.hn

anaerazomilla@gmail.com 